\title{
Ampullary Adenocarcinoma with Incidental Pancreatic Neuroendocrine Tumor: Report of an Extremely Rare Case and Review of Literature
}

\author{
Matteo Viti ${ }^{1}$, Pietro Maria Lombardi ${ }^{1}$, Mattia Marinelli ${ }^{1}$, Monica Onorati ${ }^{2}$, Corrado D'Urbano ${ }^{1}$ \\ 1. General Surgery, G. Salvini Hospital Garbagnate Milanese, Asst Rhodense, Garbagnate Milanese, ITA 2. Pathology, G. \\ Salvini Hospital Garbagnate Milanese, Asst Rhodense, Garbagnate Milanese, ITA
}

Corresponding author: Pietro Maria Lombardi, pietrom.lombardi@gmail.com

\begin{abstract}
Periampullary neoplasms are a heterogeneous group of tumors arising within $2 \mathrm{~cm}$ of the ampulla of Vater. Neuroendocrine tumors can originate throughout the entire body, from neuroendocrine cells. These neoplasms exhibit deep differences, according to their origin and biological behavior.
\end{abstract}

We describe a case of a 79-year-old man who underwent pancreaticoduodenectomy for adenocarcinoma of the ampulla of Vater after proper staging. At gross histology, an incidental pancreatic neuroendocrine tumor was also documented. Despite two synchronous neoplasms, the patient survived 34 months with no evidence of recurrence at follow-up.

The synchronous presence of a second primitive tumor in patients affected by a neuroendocrine tumor is reported in the literature; incidence is variable and the most common site is the gastrointestinal tract. Diagnostic workup for ampullary neoplasms includes abdominal computed tomography (CT) scan, magnetic resonance imaging (MRI) and endoscopic ultrasound (EUS). These investigations infrequently may detect subcentimetric lesions.

We believe this case is currently extremely rare. Preoperative diagnosis of synchronous PanNET would not have changed our approach since surgical therapy represents the gold standard in resectable ampullary neoplasms, and it has a primary role in the prognosis of the present patient.

Received 10/25/2019

Review began 11/04/2019 Review ended 11/11/2019 Published 11/13/2019

\section{๑) Copyright 2019}

Viti et al. This is an open access article distributed under the terms of the Creative Commons Attribution License CC-BY 3.0., which permits unrestricted use, distribution, and reproduction in any medium, provided the original author and source are credited.
Categories: Pathology, General Surgery, Oncology

Keywords: ampullary neoplasm, vater's ampulla adenocarcinoma, pancreaticoduodenectomy, p-nets

\section{Introduction}

Periampullary neoplasms are a heterogeneous group of tumors arising within $2 \mathrm{~cm}$ of the ampulla of Vater (AoV), including ampullary adenocarcinoma, distal cholangiocarcinoma, duodenal adenocarcinoma, and pancreatic ductal adenocarcinoma [1], having different origins and behaviors. Neuroendocrine neoplasms (NENs) can arise throughout the entire body from neuroendocrine cells; they are often found in the lungs and gastroenteropancreatic (GEP) system, less frequently in the skin, breast, and genitourinary tract [2]. A relationship exists between the presence of GEP-NENs and the simultaneous presence of primitive neoplasms in other body regions [3-4].

To the best of our knowledge, only another one case has been reported in the literature about the synchronous unknown presence of a pancreatic neuroendocrine tumor (PanNET) and AoV adenocarcinoma in the same resected specimen [5]. We herein report one case of a patient affected by these two neoplasms; PanNET diagnosis was performed by gross histology on the resected specimen after duodenopancreatectomy procedure. Moreover, we would like to underline that the detection of PanNET was an occasional finding during the pathological examination of the surgical specimen. This work has been reported in line with the Consensus Surgical Case Report (SCARE) criteria [6].

\section{Case Presentation}

A 79-year-old man was admitted to the emergency department of our hospital for abdominal pain and jaundice. He was referred to our attention by the family physician. His medical history consisted of benign prostatic hyperplasia (BPH), mild mitral regurgitation, and chronic obstructive pulmonary disease (COPD), without previous surgical procedures; he had no family history of neoplasms. He was a former smoker. On physical examination, no mass or tenderness was detected. Vital signs were normal. Blood tests showed hyperbilirubinemia ( $4.5 \mathrm{mg} / \mathrm{dl})$. Abdominal ultrasonography (US) was performed, showing dilatation of both biliary systems.

The patient was admitted to our department of general surgery in order to perform further diagnostic tests. 


\section{Cureus}

The abdominal computed tomography (CT) scan (Figure 1) revealed an important dilatation of the biliary system and major pancreatic duct (MPD). The latter and terminal part of the common bile duct (CBD) showed a blunt interruption in the pre-ampullary tract. No other lesions were observed. Tumor markers dosage revealed high levels of CA19.9 (305 U/ml, range 0-33 U/ml).

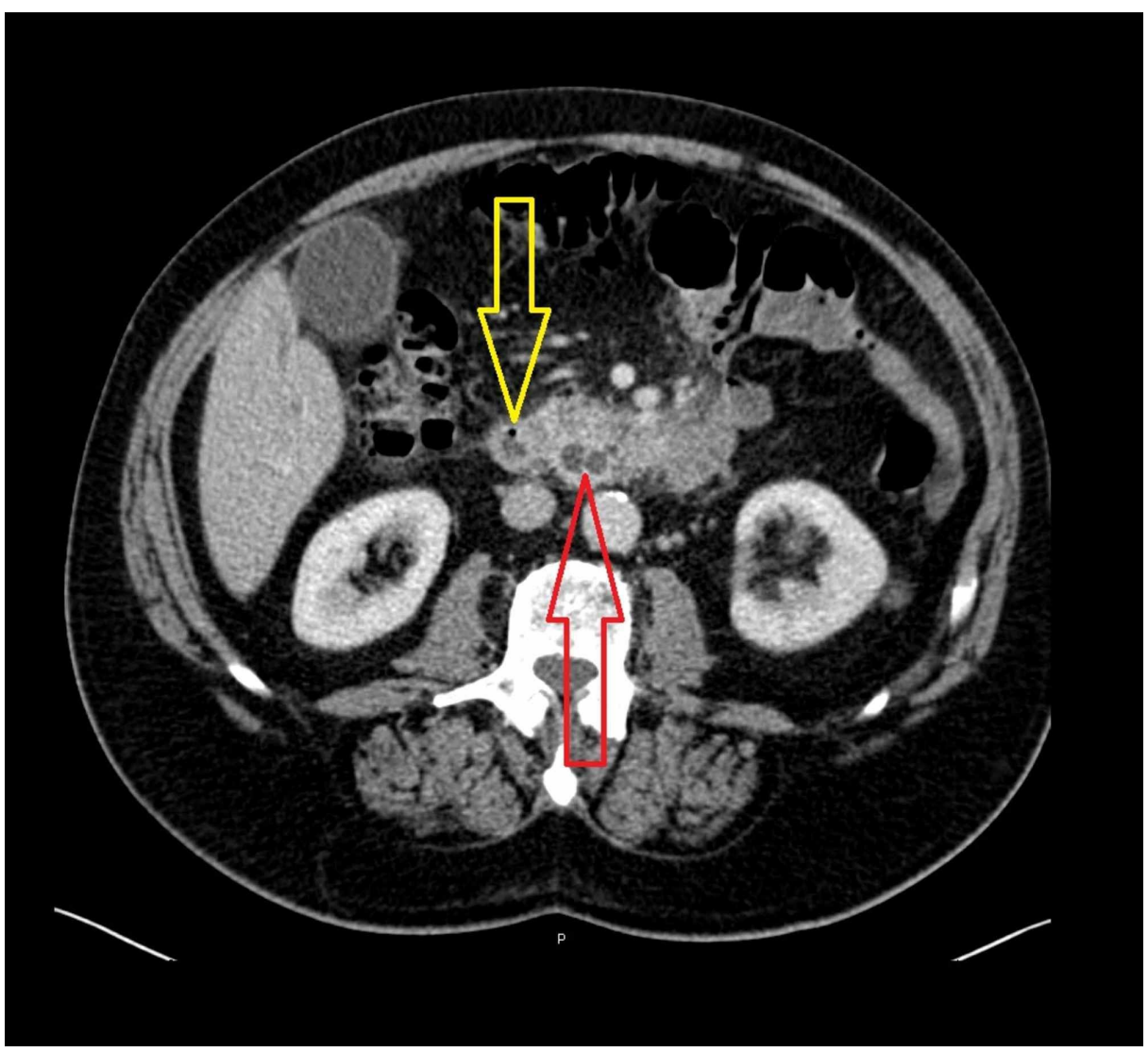

FIGURE 1: Abdominal CT scan. The red arrow indicates the dilatation of the common bile duct (CBD) and major pancreatic duct (MPD) caused by the endoluminal duodenal mass (yellow arrow).

CBD: common bile duct. MPD: major pancreatic duct

Magnetic resonance imaging (MRI) documented a 15-mm low-intensity mass, seemingly protruding in the duodenal lumen with the involvement of the major pancreatic duct (MPD) (Figures 2-3). Due to these findings, a peri-ampullary neoplasm was suspected. 


\section{Cureus}

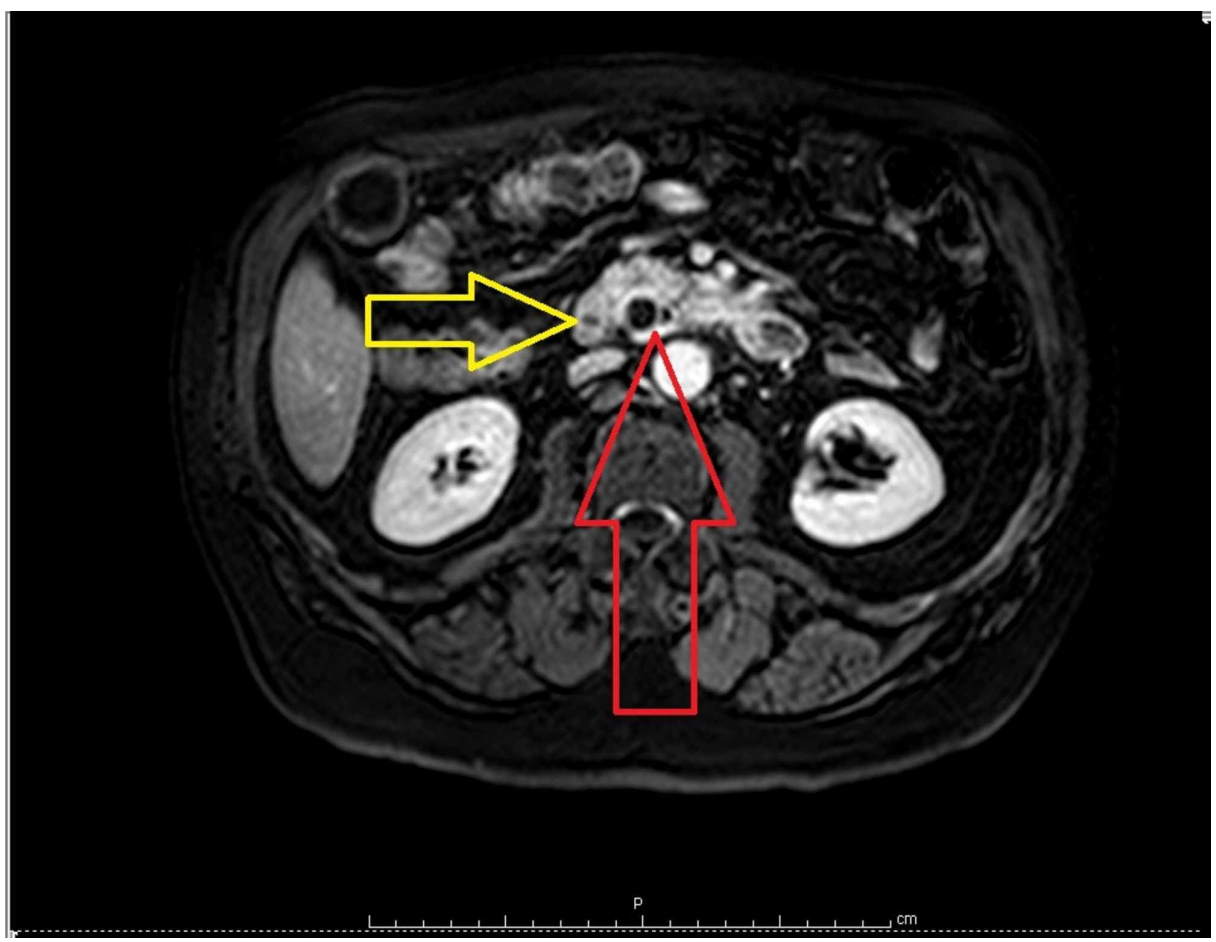

FIGURE 2: Abdominal magnetic resonance imaging (MRI). The red arrow indicates the dilatation of the common bile duct (CBD) and major pancreatic duct (MPD) caused by the endoluminal duodenal mass (yellow arrow).

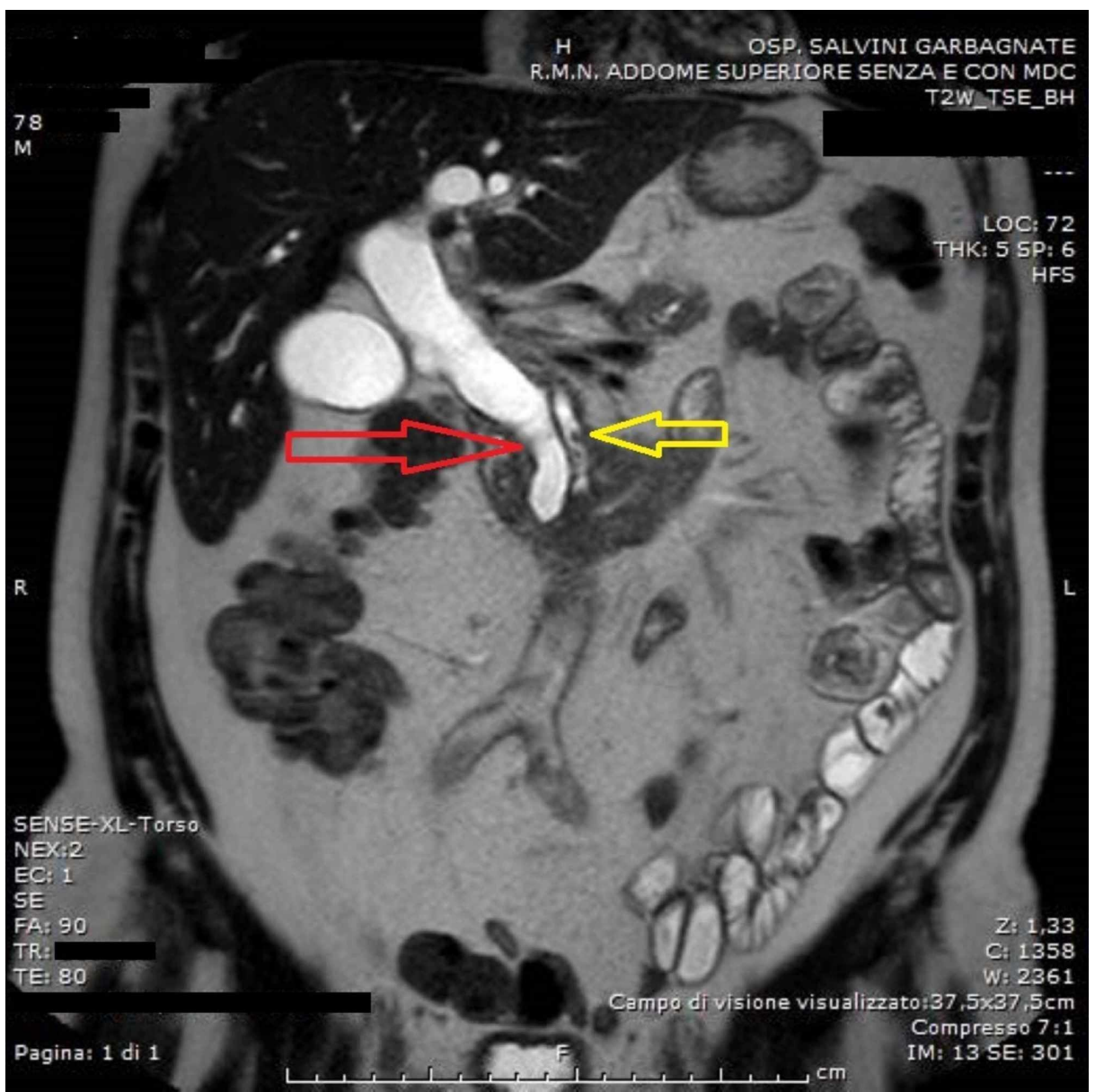


Endoscopic ultrasound (EUS) and endoscopic retrograde cholangiopancreatography (ERCP) confirmed an $18 \times 17 \mathrm{~mm}$ ipoechogenic nodular lesion close to AoV, with infiltration of the duodenal muscularis propria and pancreatic parenchyma. An enlarged lymph node was detected at the porta hepatis. During the examination, fine needle aspiration (FNA) of the ampullary lesion was performed, revealing the presence of malignant cells. After a multidisciplinary team meeting, surgery was recommended. A preoperative evaluation was performed, including a chest X-ray, and the patient underwent the Whipple procedure (pancreaticoduodenectomy) with portal and peri-choledocical lymphadenectomy. The postoperative course was uneventful, and the patient was discharged on his eleventh postoperative day in good clinical condition.

The macroscopic surgical pathology report described two distinctive neoplasms (Figure 4). The first neoplasm, detected during the radiological examination, was located at the junction of the ampulla and the duodenal mucosa and was described as a $3.5 \times 3 \mathrm{~cm}$, grayish, tender, ulcerated mass. The second neoplasm, documented for the first time during the macroscopic examination of the surgical specimen, was described as a $0.7 \mathrm{~cm}$, capsulated lesion, located $1.2 \mathrm{~cm}$ away from the ampullary adenocarcinoma, in the lumen of the minor pancreatic duct. Microscopic examination revealed, for the first lesion, an invasive, moderately differentiated, intestinal-type ampullary adenocarcinoma, infiltrating the muscularis propria. The second neoplasm was classified as panNET G2 (Ki67 index: 3\% of the neoplastic proliferation) and confirmed by immunohistochemical results (positivity to chromogranin, synaptophysin, and neuron-specific enolase (NSE)). Nine lymph nodes were examined, and all were negative for adenocarcinoma and pNET. The final pathological stage, based on the American Joint Committee on Cancer and the International Union Against Cancer (UICC TNM) staging system (8th edition), was pT2NOMx G2 for the ampullary adenocarcinoma and pT1NOMx G2 for the panNET.

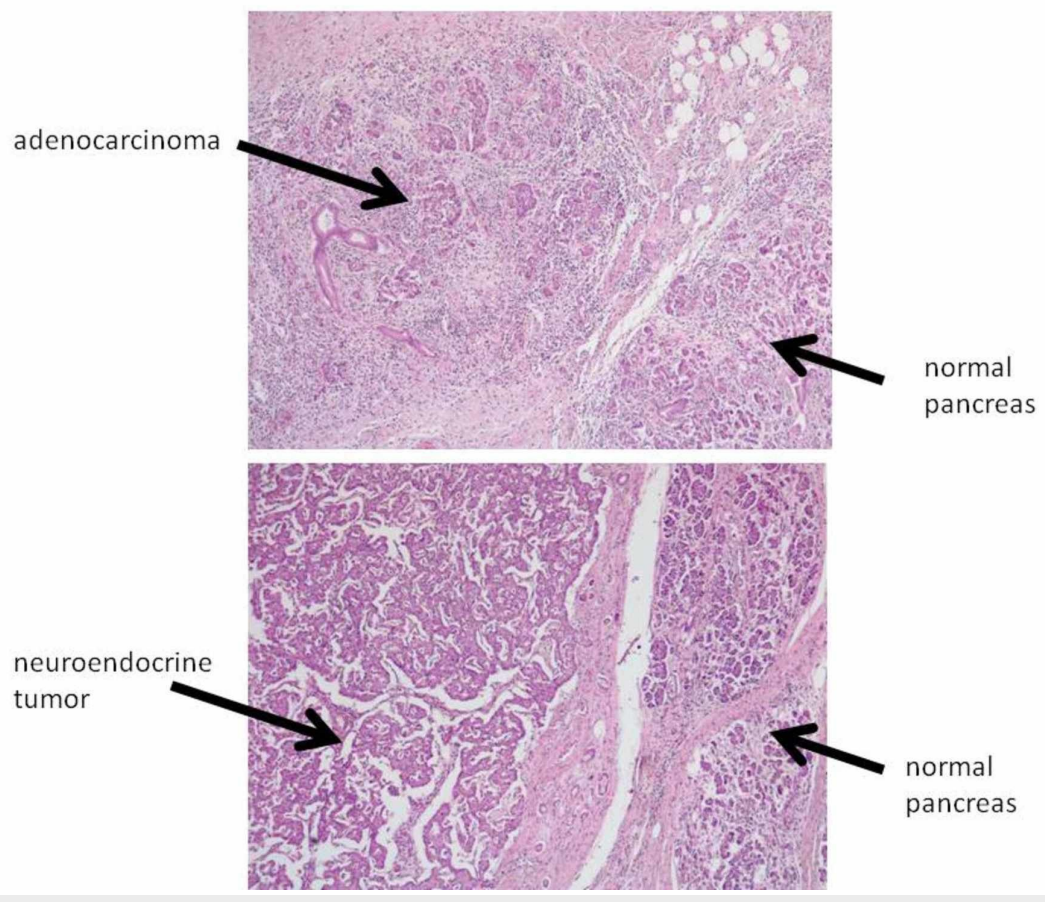

\section{FIGURE 4: Microscopic examination.}

In the upper picture, the infiltrating ductal adenocarcinoma shows disordered glandular structures intermingled with mild periglandular fibrosis (H\&E; x5). In the lower picture, the neuroendocrine pancreatic tumor shows nests of small polygonal cells, separated by thin fibrous septae and typical salt-and-pepper chromatin in each cell (H\&E; x5).

H\&E: hematoxylin and eosin stain

The patient survived 34 months after surgical treatment. He died during a hospitalization due to a urinary tract infection. Septic state was the cause of death. No evidence of disease recurrence was found at the last oncological follow-up performed two months prior that hospitalization. 


\section{Discussion}

Periampullary neoplasms and neuroendocrine neoplasms (NENs) exhibit significant differences, according to their origin and biological behavior.

The first ones are both benign and malignant lesions that arise at the inlet of the CBD and MPD, in proximity to $\mathrm{AoV}[2]$. AoV adenocarcinoma is a rare lesion, accounting for $0.2 \%$ of all malignant neoplasms of the gastrointestinal (GI) tract and almost 6\% of periampullary neoplasms [1]. Its incidence is 2.9 new cases per million/year [7]. Diagnosis relies on several clinical, radiological, and endoscopic investigations. Symptoms could include nausea, anorexia, weight loss, and abdominal pain in association with jaundice due to failed bile drainage. An abdominal CT scan allows diagnosis and staging. MRI provides an anatomical representation of the biliary tree and of the main structures of the ampullary region. EUS provides $\mathrm{T}$ staging, imaging of surrounding structures, and N staging. ERCP allows sampling and biliary stent placement when appropriate. Surgical resection can be performed with pancreaticoduodenectomy as the standard approach. In high-risk patients, local or endoscopic treatment can be offered [8]. Five-year survival rates are about $10 \%$ in pancreatic adenocarcinoma and 40\% in AoV adenocarcinoma after adequate resection [7].

NENs develop from neuroendocrine cells, which are located in several organs [2,5]. PanNETs are relatively rare entities, representing almost $1 \%-2 \%$ of whole pancreatic neoplasms [9]. PanNETs can be classified as "functioning" or "non-functioning." The latter are often asymptomatic and diagnosis is incidental [10] unless mass effect and/or metastatic disease occurs, making surgical cure impossible. The Fifth WHO has classified PanNENs into two groups, well-differentiated (neuroendocrine tumors or NET, G1-2-3) and poorly-differentiated (neuroendocrine carcinoma or NEC, G3) [11].

Preoperative diagnosis can be formulated with high accuracy and sensitivity through EUS, which can detect subcentimetric lesions with mean detection rates of over 90\% [12]. Further imaging examinations include an abdominal CT scan, MRI, and somatostatin-analogs-scintigraphy [13]. Surgical resection is mandatory for functioning lesions and for those more of $10 \mathrm{~mm}$ because in these two conditions, the risk of lymph node involvement is higher [9,12]. Five-year survival rates are about 30\% in non-functioning PanNETs and up to 97\% in surgical resectable functioning PanNETs. The synchronous presence of a second primitive tumor in patients affected by GEP-NENs is reported in the literature; incidence is variable from $12 \%$ to $46 \%$, and the most common site is the GI tract [3,14-16]. This setting may be explained by "field-effect theory," whereby a single carcinogenic agent could stimulate the development of both tumors [17-18]. Moreover, NETs produce several neuropeptides/non-neuropeptides comparable to a growth factor and could promote the development of a synchronous primitive tumor within target organs [19]. Therefore, investigating the presence of a synchronous primitive tumor in patients affected by NET is recommended [3].

In the present case, the patient came to our attention with a diagnosis of adenocarcinoma of AoV, and he underwent proper staging by abdominal CT scan, MRI, and EUS. Those investigations did not raise suspicion of any other lesions.

\section{Conclusions}

In conclusion, we believe that the present case is extremely rare since we found only another one case in the literature regarding the synchronous presence of PanNET and AoV adenocarcinoma. Despite proper preoperative workup, PanNET was reported only on histological examination of the surgical specimen. In the present case, according to our experience, a preoperative diagnosis of synchronous PanNET would not have changed our approach. Surgical therapy represented the gold standard treatment and played a primary role in the patient's prognosis.

\section{Additional Information \\ Disclosures}

Human subjects: Consent was obtained by all participants in this study. Conflicts of interest: In compliance with the ICMJE uniform disclosure form, all authors declare the following: Payment/services info: All authors have declared that no financial support was received from any organization for the submitted work. Financial relationships: All authors have declared that they have no financial relationships at present or within the previous three years with any organizations that might have an interest in the submitted work. Other relationships: All authors have declared that there are no other relationships or activities that could appear to have influenced the submitted work.

\section{References}

1. Okano K, Oshima M, Yachida S, et al.: Factors predicting survival and pathological subtype in patients with ampullary adenocarcinoma. J Surg Oncol. 2014, 110:156-162. 10.1002/jso.23600

2. National Comprehensive Cancer Network. NCCN clinical practice guidelines in oncology: neuroendocrine and adrenal tumors. (2018). Accessed: December 23, 2018:

https://www.nccn.org/professionals/physician_gls/default.aspx.

3. Parra-Medina R, Moreno-Lucero P, Jimenez-Moreno J, Parra-Morales AM, Romero-Rojas A: Neuroendocrine 
neoplasms of gastrointestinal tract and secondary primary synchronous tumors: a systematic review of case reports. Casualty or causality?. PLoS One. 2019, 2019:0216647. 10.1371/journal.pone.0216647

4. Tavares AB, Viveiros FA, Cidade CN, Maciel J: Gastric GIST with synchronous neuroendocrine tumour of the pancreas in a patient without neurofibromatosis type 1. BMJ Case Rep. 2012, 2012:bcr0220125895. 10.1136/bcr.02.2012.5895

5. Sastry A, Wayne M, Steele J, Iskandar M, Yuan S, Cooperman AM: Three synchronous, sporadic and separate periampullary and pancreatic tumors: more than a coincidence?. World J Surg Oncol. 2014, 2014:382. 10.1186/1477-7819-12-382

6. Agha RA, Borrelli MR, Farwana R, Koshy K, Fowler A, Orgill DP, the SCARE Group: The SCARE 2018 Statement: updating Consensus Surgical CAse REport (SCARE) guidelines. Int J Surg. 2018, 60:132-136 10.1016/j.ijsu.2018.10.028

7. Prenzel KL, Hölscher AH, Drebber U, Bollschweiler E, Gutschow C, Stippel D, Mönig S: Extracapsular lymph node spread as a negative prognostic factor of adenocarcinoma of the pancreas and cancer of the papilla of Vater. Pancreas. 2014, 43:64-68. 10.1097/MPA.0b013e3182a44a91

8. Gaspar B, Beuran M, Paun S, Ganescu R, Hostiuc S, Negoi I: Current strategies in the therapeutic approach for adenocarcinoma of the ampulla of Vater. J Med Life. 2013, 6:260-265.

9. Halfdanarson TR, Rubin J, Farnell MB, Grant CS, Petersen GM: Pancreatic endocrine neoplasms: epidemiology and prognosis of pancreatic endocrine tumors. Endocr Relat Cancer. 2008, 15:409-427.

10. Kim MK: Endoscopic ultrasound in gastroenteropancreatic neuroendocrine tumors . Gut Liver. 2012, 6:405410. 10.5009/gnl.2012.6.4.405

11. Inzani F, Petrone G, Rindi G: The new World Health Organization classification for pancreatic neuroendocrine neoplasia. Endocrinol Metab Clin North Am. 2018, 47:463-470. 10.1016/j.ecl.2018.04.008

12. Khashab MA, Yong E, Lennon AM, et al.: EUS is still superior to multidetector computerized tomography for detection of pancreatic neuroendocrine tumors. Gastrointest Endosc. 2011, 73:691-696. 10.1016/i.gie.2010.08.030

13. Jian S: Pancreatic neuroendocrine tumors. Intractable Rare Dis Res. 2017, 6:21-28. 10.5582/irdr.2017.01007

14. Kishi Y, Shimada K, Nara S, Esaki M, Hiraoka N, Kosuge T: Basing treatment strategy for non-functional pancreatic neuroendocrine tumors on tumor size. Ann Surg Oncol. 2014, 21:2882-2888. 10.1245/s10434014-3701-y

15. Cokmert S, Demir L, Akder Sari A, et al.: Synchronous appearance of a high-grade neuroendocrine carcinoma of the ampulla Vater and sigmoid colon adenocarcinoma. Case Rep Oncol Med. 2013, 2013:930359. 10.1155/2013/930359

16. Pearson CM, Fitzgerald PJ: Carcinoid tumors - a re-emphasis of their malignant nature. Review of 140 cases . Cancer. 1949, 2:1005-1026. 10.1002/1097-0142(194911)2:6<1005::AID-CNCR2820020608>3.0.CO;2-1

17. Prommegger R, Ensiger C, Steiner P, Sauper T, Profanter C, Margreiter R: Neuroendocrine tumors and second primary malignancy - a relationship with clinical impact?. Anticancer Res. 2004, 24:1049-1051.

18. Zuker KA, Longo WE, Modlin IM, Bilchik AJ, Adrian TE: Malignant diathesis from jejunal-ileal carcinoids . Am J Gastroenterol. 1989, 84:182-186.

19. Oberg K: Expression of growth factor and their receptors in neuroendocrine gut and pancreatic tumors, and prognostic factors for survival. Annals of the New York Academy of Science. 1994, 733:46-55. 10.1111/j.1749-6632.1994.tb17255.x 